biopsy. Treatment by chemotherapy and/or irradiation was successful. At follow-up of 12 to 18 months, patients were in remission. Patients 1 and 2 were dependent on thyroxin, hydrocortisone, and growth hormone, while patient 3 needed no further hormone substitution. (Gottschling S, Graf N, Meyer S, et al. Intracranial germinoma: a rare but important differential diagnosis in children with growth retardation. Acta Paediatrica March 2006;95:302-305). (Respond: Dr Sven Gottschling, University Children's Hospital, Kirrbergerstr, 66421 Homburg/Saar, Germany).

COMMENT. The incidence of pediatric intracranial germ cell tumors varies worldwide, the average being $3.6 \%$, and higher in Asia than the Western hemisphere. Germinomas arise in the pineal or suprasellar regions and if diagnosed early, have a good prognosis. The present small series demonstrates the value of secondary growth retardation as an early sign of suprasellar germinoma. Diagnosis is made by MRI, and a CSF B-hCG level above $50 \mathrm{U} / 1$ (under $7 \mathrm{U} / \mathrm{I}$ is normal). Biopsy may be necessary when the hormone levels are not elevated.

A larger series of 26 children with germ cell tumors (17 germinomas and 9 teratomas), not cited above, was reported from the University Hospital Hamburg (Haupt $\mathrm{C}$ et al. Eur J Pediatr 1996;155:230-236; Ped Neur Briefs May 1996). Tumor location was the pineal region in $69 \%$ and suprasellar/hypothalamic in $31 \%$. Presenting symptoms were increased intracranial pressure, Parinaud's syndrome, and endocrine deficits. Long-term survival rate was $88 \%$ for germinomas and $43 \%$ for malignant teratomas.

\title{
ENCEPHALOPATHIES
}

\section{ACUTE INFANTILE FRONTAL LOBE ENCEPHALOPATHY}

The clinical and radiologic features of nine infants with acute encephalopathy involving the frontal lobes are reported from Dokkyo University School of Medicine and Jichi Medical School, Tochigi; and University of Tokyo, Japan. The prenatal and perinatal histories were uneventful, and milestones of development were normal. Symptoms presented at 7 months to 3 years of age with 1) convulsive status epilepticus with hyperpyrexia and prolonged impairment of consciousness, 2) mental and motor regression after recovery of concciousness, 3) CSF leukocyte count of $8 \mathrm{cells} / \mathrm{mm}^{3}$, 4) MRI showing atrophy of both frontal lobes. The infection was influenza type A in 3 patients, exanthem subitum in 2, measles in 1, and upper respiratory viral illness nonspecified in 3 . Regression of behavior on recovery of consciousness involved verbal function and language, stereotypic movements in 4 , and cataplexy in 1. On 12-month follow-up, motor function recovered while speech remained retarded. Laboratory tests revealed increased lactate dehydrogenase and creatine kinase, normal CSF protein in all except one patient, and elevated interleukin- 6 in 2 of 3 tested. EEG at time of onset showed high-amplitude delta predominantly in both frontal areas, and at 1 year, focal spikes or spike-and-wave discharge in 3 patients. One patient developed localization-related epilepsy. On serial MRI performed between the $20^{\text {th }}$ and $42^{\text {nd }}$ day from onset, atrophic changes were revealed in both frontal lobes of all patients. PET studies in the same period showed decreased perfusion in both frontal lobes, with normalized perfusion at the $7^{\text {th }}$ to 38 th month after onset. (Yamanouchi H, Kawaguchi N, Mori M, et al. Acute infantile encephalopathy predominantly affecting the frontal lobes. Pediatr Neurol 
February 2006;34:93-100). (Respond: Dr Yamanouchi, Department of Pediatrics, Dokkyo University School of Medicine, 880 Kitakobayashi, Mibu, Shimotsuga, Tochigi 321-0293, Japan).

COMMENT. The differentiation of acute encephalopathy and complex febrile seizure (CFS) is often difficult, and the clinical presentation of all 9 infants with prolonged seizures and high fever highlights this controversy. Factors in favor of the diagnosis of CFS in the above series of patients include the type of infection causing the fever and seizure (influenza A, exanthem subitum, and nonspecific viral URI, all commonly associated with FS); and the elevation of interleukin- 6 cytokines, previously reported in children with FS and with influenza encephalopathy (Virta M et al. Epilepsia 2002;43:920-923; Kawada J-I et al. J Infect Dis 2003;188:690-698). The role of the cytokine response to infection in relation to the mechanism of febrile seizures is discussed in subsequent correspondence (Millichap JG, Millichap JJ. J Infect Dis 2004;189:564-565). A previous study negating an association of elevated cytokines and FS (Ichiyama T et al. Neurology 1998;50:407-411), cited by the authors of the current report, is now discounted.

The prolonged impairment of consciousness after the convulsions, and the behavioral sequelae are consistent with an encephalopathy. These cases are unique in the predilection for frontal lobe involvement, a finding that differentiates them from other acute encephalopathies of childhood, such as Reye syndrome and acute disseminated encephalomyelitis, but does not exclude a postictal pathology.

A rare case of acute necrotizing encephalopathy (ANE) associated with human herpesvirus-6 infection is reported from Sapporo, Japan (Ohsaka M et al. Pediatr Neurol Feb 2006;34:160-163). A 14-month-old infant presented with high fever and generalized tonic convulsion followed by persistent coma, rash of exanthem subitum on the $4^{\text {th }}$ day, brain swelling and herniation, the patient dying on the $12^{\text {th }}$ day of the illness. CSF protein was 73 $\mathrm{mg} / \mathrm{dL}$, cell count 2 , glucose $155 \mathrm{mg} / \mathrm{dL}$. The MRI on the $3^{\text {rd }}$ day revealed symmetric lesions in the thalamus, cerebellum, and brainstem, typical of ANE.

Cortical liquefaction is reported in a 9-month-old infant with severe HHV-6 encephalopathy (Takanashi J et al. Neurology Feb (1 of 2) 2006;66:452). MRI showing high signal intensity on T2-weighted images and low signal intensity on T1-weighted and FLAIR images in the cortex was consistent with liquefaction. Consciousness gradually improved, but the infant had severe retardation and quadriplegia.

\section{ALEXANDER DISEASE WITHOUT WHITE MATTER LESIONS}

Seven patients with atypical Alexander disease, showing signs of medulla and spinal cord involvement and little evidence of leukoencephalopathy, are reported from University Medical Center, Amsterdam, the Netherlands; and centers in the UK, USA, Canada, and Sweden. Patient 1 presented with progressive spinal cord dysfunction at age 9 years. Spinal MRI showed thickening of the spinal cord, and brain MRI revealed medulla and dentate nucleus changes, with a garland appearance lining the lateral ventricles. Rosenthal fibers consistent with Alexander disease were found in perivascular and subependymal regions of the ventricular wall biopsy. Diagnosis was confirmed by genetic testing and de novo mutations in the GFAP gene. All patients had juvenile onset and signs of brain stem and 